
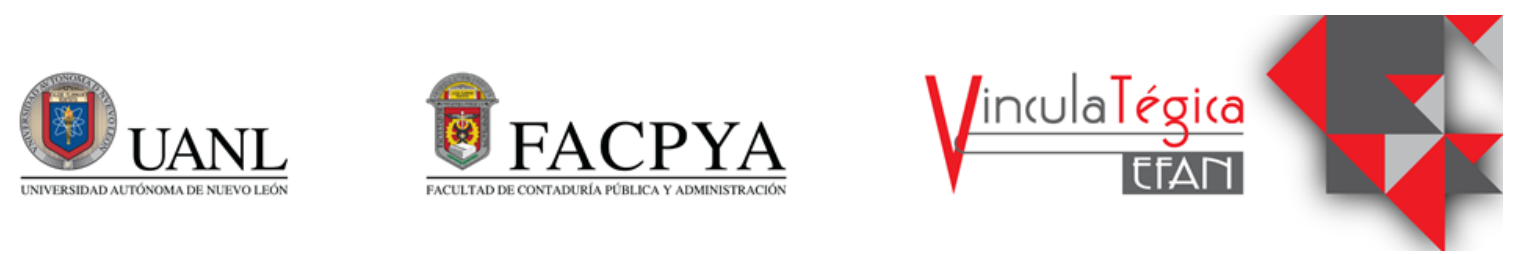

\title{
El entorno personal de aprendizaje como estrategia asociada con el desarrollo de habilidades de autogestión del aprendizaje en estudiantes de licenciatura en administración
}

\author{
Alma Elizabeth Merla González ${ }^{1}$, María Magdalena Madrigal Lozano² y Claudia Dörfer ${ }^{3}$ \\ ${ }^{1}$ Escuela de Ciencias de la Educación, amerla@ece.edu.mx, Serafín Peña \#130 sur, Centro Monterrey, \\ Nuevo León, México, 52(81)8340-3612 \\ ${ }^{2}$ Universidad Autónoma de Nuevo León, maria.madrigallz@uanl.edu.mx, Pedro de Alba s $/ n$ San Nicolás de \\ los Garza, Nuevo León, México, 52(81)8329-4000 \\ ${ }^{3}$ Universidad Autónoma de Nuevo León, claudia.doerfer@uanl.edu.mx, Pedro de Alba s/n San Nicolás de los \\ Garza, Nuevo León, México, 52(81)8329-4000
}

Información del artículo revisado por pares

Fecha de aceptación: junio-2021

Fecha de publicación en línea: diciembre-2021

DOI:https://doi.org/10.29105/vtga7.1-92

\section{Resumen}

Esta investigación se enfoca en el tema de los Entornos Personales de Aprendizaje o Personal Learning Enviroment (PLE), como estrategia pedagógica asociada con el desarrollo de habilidades metacognitivas de autorregulación y autonomía que permiten al estudiante autogestionar su aprendizaje en un entorno virtual. Desde esta perspectiva, se realizó un estudio no experimental, transaccional y de alcance descriptivo para conocer los componentes del PLE (estrategias, procesos y tecnologías) que utiliza el estudiante, para desarrollar sus habilidades metacognitivas de autogestión del aprendizaje, como procesos convenientes para cumplir los propósitos de formación de una Unidad de Aprendizaje del programa de Licenciatura en Administración, impartida en modalidad virtual en contexto de pandemia por COVID-19, por una universidad pública del Noreste de México. En el proceso participaron 42 estudiantes inscritos en el semestre febrero - julio de 2021. El análisis de los datos se realizó mediante pruebas no paramétricas para generar resultados descriptivos sobre las herramientas digitales del PLE del estudiante en el desarrollo de sus procesos de autogestión del aprendizaje, particularmente en acciones relacionadas con la autogestión de la información. Los hallazgos muestran que los recursos tecnológicos que emplean los estudiantes en la configuración de su PLE, marcan una alta tendencia

\section{Abstract}

This research focuses on the topic of Personal Learning Environments (PLE) as a pedagogical strategy associated with the development of metacognitive skills of self-regulation and autonomy that allow the student to self-manage their learning in a virtual environment. From this perspective, a non-experimental, transactional study with a descriptive scope was carried out to know the components of the PLE (strategies, processes and technologies) that the student uses, to develop their metacognitive skills of selfmanagement of learning, as convenient processes to fulfill the purposes of a Learning Unit of the Bachelor of Administration program, taught in virtual mode in the context of a COVID-19 pandemic, by a public university in Northeast Mexico. 42 students enrolled in the semester February - July 2021 participated in the process. The data analysis was carried out using nonparametric tests to generate descriptive results on the digital tools of the student's PLE in the development of their learning self-management processes, particularly in actions related to the selfmanagement of information. The findings show that the technological resources that students use in the configuration of their PLE, mark a high trend towards audiovisual media to strengthen their metacognitive skills of autonomy and self- 
hacia los medios audiovisuales para fortalecer sus habilidades metacognitivas de autonomía y autogestión del aprendizaje.

Palabras clave: entorno Personal de Aprendizaje, herramientas digitales, educación virtual, metacognición, autogestión del aprendizaje.

\section{INTRODUCCIÓN}

El presente artículo muestra los hallazgos de un estudio centrado en analizar la configuración del Entorno Personal de Aprendizaje o Personal Learning Enviroment (PLE, por sus siglas en inglés), como estrategia pedagógica emergente asociada con el desarrollo de habilidades metacognitivas de autorregulación y autonomía que permiten al estudiante de un programa de licenciatura autogestionar su aprendizaje en un entorno virtual (Dabbagh y Kitsantas, 2012; Tarancón, 2017; García y González, 2019). Al momento, dicho concepto ha tomado relevancia en el ámbito educativo, como un tema de estudio que demanda encontrar explicaciones acerca de cómo aprenden las personas en un mundo conectado por la tecnología. De acuerdo con Gallego y Chaves (2014), “el interés ha derivado hacia la relación entre el 'ambiente de aprendizaje' y lo 'personal' para tratar de describir e interpretar qué, cómo y con qué trabaja el individuo que aprende con TIC" (p. 2). En el caso de esta investigación, fue determinante identificar las estrategias, procesos y tecnologías que utilizan los estudiantes de un curso de licenciatura para lograr los objetivos programáticos de una unidad de aprendizaje gestionada en un entorno virtual. Y bajo este planteamiento, emergió la necesidad de comprender la asociación que dicho término posee con el desarrollo de las habilidades metacognitivas, debido a que, por definición, en la configuración del PLE se integran estrategias cognitivas y actitudes que promueven el aprendizaje autónomo y autorregulado en las personas, tal y como lo señalan Castañeda y Adell (2013).

Además, cabe hacer mención que ante la escasez de estudios empíricos que relacionen al PLE como un sistema que promueve el aprendizaje autorregulado en el management of learning.

Keywords: personal learning environment, digital tools, virtual education, metacognition, selfmanagement of learning.

JEL: D83, I21, I23, O32 у O33.

estudiante, prevalece el reto de analizar el uso educativo de los recursos digitales en estos entornos, desde un enfoque pedagógico que reconozca el papel activo y proactivo del estudiante en la construcción de su PLE para desarrollar la capacidad de autorregular su aprendizaje y cumplir sus objetivos académicos y personales (Ortiz, Maroto y Agreda, 2017; Meza-Cano, Morales y Flores, 2016).

Partiendo de esta idea, esta investigación se propuso identificar los componentes del PLE (estrategias, procesos y tecnologías) del estudiante de licenciatura asociados con el desarrollo de habilidades para el aprendizaje autorregulado para cumplir los objetivos de un curso gestionado a distancia, en un entorno virtual de aprendizaje (EVA), en el marco de la pandemia por COVID-19. En el entendido que los componentes que configuran el PLE del estudiante, se incluyen:

- Estrategias para ordenar y gestionar la información.

- Estrategias de autorregulación para cumplir objetivos de preparación, crear recursos, y evaluar su proceso de aprendizaje.

- Estrategias para desarrollar habilidades metacognitivas, de previsión, actuación y reflexión del proceso de autorregulación del aprendizaje.

Los componentes descritos corresponden a las dimensiones señaladas por autores que comparten el enfoque pedagógico del PLE como una estrategia que posibilita que el estudiante adquiera mayor control sobre lo que aprenden y cómo lo aprenden (Gallego y Chaves, 2014, p. 5). $\quad \mathrm{Al}$ respecto, García y González (2019), señalan la importancia de analizar los PLE de los estudiantes, debido a que posibilitan la comprensión del proceso de aprendizaje que realiza el estudiante de nivel 
superior, y conocer las herramientas tecnológicas que utiliza para cumplir los propósitos y actividades que le proponen en su trayecto formal de formación en la universidad, así como en otros espacios de formación no formal (p.31).

En esta línea, este estudio pretende analizar este fenómeno desde una conceptualización pedagógica del PLE, como un enfoque innovador en propuestas formativas mediadas por TIC, donde toma lugar el protagonismo del estudiante como eje del proceso de enseñanza-aprendizaje, constituyéndose como una propuesta compatible con los retos que demandan solventar los modelos educativos en el marco de la transformación digital que atraviesan las Instituciones de Educación Superior a raíz de la pandemia por COVID-19, entre las cuales sobresale la necesidad de "modificar el proceso de enseñanza dependiendo de las habilidades cognitivas individuales de los estudiantes" (Cabero y Llorente, 2020, p. 29).

Sin embargo, cabe mencionar que para lograr un cambio de este nivel, es importante reconocer que los estudiantes requieren fortalecer las competencias digitales para el uso y aplicación de recursos tecnológicos con fines académicos y labores relacionadas con su ámbito profesional; por tanto, los expertos en el tema recomiendan dejar al margen las ideas preestablecidas que prevalecen en los contextos educativos acerca de dar por hecho que los estudiantes dominan tales herramientas, al ser "nativos digitales" dada su condición de contemporaneidad con la evolución de era digital.

En este contexto, los investigadores del tema han reportado la necesidad de implementar propuestas educativas de alfabetización informacional que apoyen al estudiante universitario a fijar los propósitos y alcances de sus procesos de aprender, conocer y mantener la curiosidad, a resolver problemas de información y comunicación y a generar estrategias para desenvolverse con ética y responsabilidad en los espacios digitales (Chiecher y Melgar, 2018; Recio, Silva y Abricott, 2020).

Como se observa, en los contextos educativos de nivel superior surge como condición básica, la implementación de estrategias pedagógicas que promuevan el uso innovador de las herramientas tecnológicas con propósitos formativos entre los estudiantes, las cuales, sin duda, utilizan con frecuencia con otras finalidades. Por lo cual, resulta necesario que los docentes planifiquen un proceso de instrucción que aporte las condiciones idóneas para que los estudiantes desarrollen procesos de autorregulación de su aprendizaje, dado que este proceso se asume como "un factor clave que les permitirá desenvolverse en los entornos de formación a distancia" (Cabero y Llorente, 2020, p. 30).

Considerando lo descrito, la literatura señala que toda propuesta educativa actual, establezca entre sus objetivos primordiales, la importancia de desarrollar las habilidades digitales de los estudiantes, además de implementar estrategias pedagógicas innovadoras que promuevan la participación activa y consciente del estudiante sobre su proceso de instrucción, sobre todo en lo que corresponde a su capacidad para identificar las estrategias que implementa para planificar sus metas y lograr sus objetivos de aprendizaje, la forma en que toma sus decisiones, controla sus procesos cognitivos, y revisa y evalúa su desempeño durante el trayecto de su formación académica.

\section{MARCO TEÓRICO}

En el ámbito del e-learning, aproximadamente hace una década, se promovió la idea de concebir al PLE como un término asociado a un cambio significativo en los enfoques pedagógicos orientados a fortalecer el aprendizaje en ambientes virtuales, considerando que cada persona aplica los recursos tecnológicos que considera útiles para satisfacer sus necesidades relativas a su trabajo personal y rutina de aprendizaje (Attwel, 2007; Downes, 2007; Milligan, Beauvoir, Johnson, Sharples, Wilson y Liber, (2006).

Partiendo de esta perspectiva, la importancia del PLE se identificó como un concepto vinculado al ámbito pedagógico en el marco de la primera conferencia internacional dedicada al tema, con el propósito de difundir los avances de la 
literatura científica en lo concerniente a esta cuestión. (Hernández, 2016). En este evento, se otorgó notoriedad al PLE como un cambio cualitativo en lo relativo a modelos educativos, al identificarle como un término asociado con la transición que realizan los estudiantes cuando dejan de ser consumidores de información, como una situación relacionada con aspectos de dominio tecnológico, para convertirse en gestores de información y creadores de contenidos para crear conexiones significativas entre los distintos recursos tecnológicos que seleccionan y utilizan para cumplir sus propósitos formativos o de interacción social como una situación asociada con el desarrollo de habilidades metacognitivas y de autorregulación del aprendizaje. (The PLE Conference 2010, Disponible en https://pleconference.citilab.eu/cas/index.htm $1)$.

No obstante lo señalado, Castañeda y Adell (2013) ubican el uso de este término desde el año 2001, dentro de las actividades del proyecto denominado NIMLE (Northern Ireland Integrated Managed Learning Environment) financiado a su vez por el JISC (Joint Information Systems Committee) de la Gran Bretaña, como una propuesta asociada con la configuración de un entorno de aprendizaje centrado en el alumno, en contraparte a la idea ya consolidada de un entorno virtual de enseñanza-aprendizaje centrado en la institución, como gestora del proceso formativo. A su vez agregan que la utilización formal de las siglas que identifican a este concepto, se dio dentro de un congreso anual de la citada JISC, en el año 2004. A partir de entonces, se utiliza el acrónimo PLE, para identificar este término con "una idea pedagógica acerca de cómo aprenden las personas con tecnología" (Castañeda y Adell, 2013, p. 13).

Cabe destacar que el tratamiento de este término tuvo una importante evolución antes de identificarle como una estrategia pedagógica emergente de apoyo a los procesos formales en la gestión de programas académicos en modalidad virtual. $\mathrm{Su}$ conceptualización desde esta perspectiva, tomó tiempo luego de una serie de debates entre expertos e investigadores que perfilaron su definición actual, donde sobresalen sus características pedagógicas y no se le identifica como un concepto tecnológico. Es decir, en la actualidad se le identifica como una estrategia pedagógica que necesariamente, requiere del uso de tecnología. De esta forma, un Entorno Personal de Aprendizaje (PLE, por sus siglas en inglés), “...es el conjunto de herramientas, fuentes de información, conexiones y actividades que cada persona utiliza de forma asidua para aprender" (Adell y Castañeda, 2010, pág. 23).

Ahora bien, toda vez que el concepto PLE incursionó en el ámbito educativo, autores como Pane, Steiner, Baird y Hamilton, (2015) opinan que la investigación futura debería explorar buenas combinaciones de tecnología y pedagogía. En esta línea, el estudio de los PLE con fines educativos, se posicionó "como una construcción conceptual teórico-pedagógica, creada por una comunidad de profesionales en tecnologías y docentes que buscan interpretar el impacto de las tecnologías web 2.0 en el aprendizaje de las personas" (Chaves y Sola, 2018, p. 3).

Sin duda, los avances tecnológicos y el surgimiento de nuevas formas de interactuar con tales herramientas, configuraron un campo fértil para investigar las posibilidades que aporta la llamada "ecología del aprendizaje", donde se parte de la idea de cómo el estudiante integra las experiencias, procesos y estrategias que le brindan las aplicaciones y servicios de la Web 2.0, al tiempo que las integra con las experiencias adquiridas en modelos de aprendizaje tradicional. En este sentido, resulta un tema interesante para la investigación educativa, analizar las estrategias que aporta el estudiante desde la configuración de su PLE para alcanzar las metas de aprendizaje individual y colectivo que le proponen los trayectos formativos sustentados en paradigmas pedagógicos que incluyen la implementación de estrategias digitales.

\subsection{La transformación digital y su incursión en los contextos educativos} Actualmente se perfila la revolución tecnológica derivada de la industria 4.0, lo que 
representa el establecimiento de nuevos retos por solventar en el ámbito educativo, particularmente en temas de gestión y diseño de propuestas pedagógicas innovadoras que impulsen el fortalecimiento de las competencias digitales de los estudiantes para resolver problemas de su ámbito profesional mediante la aplicación de recursos tecnológicos de última generación, como lo es el Internet de las cosas, la inteligencia artificial, el intercambio de datos en tiempo real y el aprendizaje automatizado, entre otros. De esta perspectiva, los programas ofertados por las IES, habrán de sustentarse en modelos educativos de enfoque integral que "habilita a los educandos para tomar decisiones fundamentadas y adoptar medidas responsables en favor de la integridad del medio ambiente y la viabilidad de la economía" (Disponible en www.unesco.org). Lo descrito toma lugar desde la visión de una educación para el desarrollo sostenible (EDS) como un proceso de aprendizaje permanente que tiene como propósito "desarrollar los conocimientos, las habilidades, los valores y las actitudes que nos permitan a todos tomar decisiones fundamentadas y adoptar medidas individuales y colectivas sobre las cuestiones urgentes a nivel local, nacional y mundial" (UNESCO, 2020, p. 8).

En este escenario, el PLE como una propuesta pedagógica emergente pudiese aportar elementos para cumplir los propósitos de la Educación para el Desarrollo Sustentable (EDS), propuestos por la UNESCO en ámbito de acción prioritario 2, Transformación de los Entornos de Aprendizaje; que establece la importancia de promover las condiciones idóneas para que los estudiantes "aprendan lo que viven y vivan lo que aprenden” (p.28). Lo cual coincide con lo señalado por Boulahrouz, Medir y Calabuig (2019) en el sentido de que "la continua expansión de las tecnologías junto con el aumento de la familiaridad de los jóvenes con las herramientas, aplicaciones de redes sociales, y la aceptación de métodos pedagógicos innovadores en el sistema educativo ofrece nuevas posibilidades para la EDS" (p. 83).

Dicho lo anterior, se justifica el interés por analizar el potencial y las ventajas que aportan las estrategias pedagógicas emergentes, como es el caso del PLE, en la implementación de procesos de instrucción en entornos virtuales. Aunado a los avances logrados en la conceptualización del PLE, en el ámbito disciplinar de la Tecnología Educativa, la investigación actual ha generado resultados relevantes para comprender el tema. Es así como encontramos estudios centrados en analizar las dimensiones que componen un PLE, como ha sido reportado en la literatura, a saber: dimensión relacionada con estrategias para ordenar y gestionar la información (Prendes, Román y González, 2019); Leiva, Cabero y Ugalde, 2018). Dimensión acerca de estrategias de autorregulación para cumplir metas de aprendizaje, para crear y compartir con otros, (García y González, 2019; Ortiz-Colón, Maroto y Agreda, 2017). Y, dimensión relativa a las estrategias de actuación y reflexión que hacen posible el desarrollo de habilidades metacognitivas (Chaves, Trujillo y López, 2015; Calle-Álvarez y Sánchez-Castro, 2017).

En particular, los estudios de López, González, Aguiar y Artiles, (2017), Prendes, Román y González, (2019), Leiva, Cabero y Ugalde, (2018) aportan hallazgos para conocer las peculiaridades de la configuración de estos entornos y los tipos de estrategias que utilizan los estudiantes universitarios para construirlos e implementar dichas estrategias en procesos de educación formal. Además de reportar información sobre la utilización de las herramientas tecnológicas de sus PLE en aspectos relacionados con la gestión de la información, cómo la busca, filtra y selecciona, cómo la organiza, cómo la combina para generar nueva información, y cómo la comunica y la comparte a otros. Por otro lado, se encuentran estudios sobre variables individuales relacionadas con la construcción y uso de un Entorno Personal de Aprendizaje (Meza, Morales y Flores, 2016); así como investigaciones sobre el PLE como una propuesta de creación propia para seleccionar aplicaciones de la Web 2.0 que complementen el aprendizaje autónomo de estudiantes del Grado de Administración y Dirección de Empresas (González y Calvo, 
2019).

A lo anterior, se suman los estudios enfocados en "identificar las características de los PLE de los estudiantes para sobre esa base rediseñar la práctica pedagógica y contribuir al desarrollo de las competencias necesarias para el autoaprendizaje permanente de los estudiantes" (Jerez y Barroso, 2020), p. 205). Además de investigaciones que reportan cómo el estudiante, diseña, construye y evalúa su propio PLE, con la finalidad de cumplir las actividades que le demanda el trayecto formativo de una asignatura relacionada con las tecnologías aplicadas a la educación (Chaves-Barboza y Sola-Martínez, 2018). Por último, se encuentran las tendencias en investigación que pretenden resolver situaciones relacionadas con las ecologías de aprendizaje en la era digital, como es el caso del estudio de García y González (2020), donde se destaca la necesidad de estudiar el desarrollo de las competencias comunicativas como componentes clave de los entornos personales de aprendizaje (PLE).

\subsection{El concepto PLE y su asociación con procesos de educación flexible para el desarrollo de habilidades de autogestión del aprendizaje en el estudiante}

En su momento, autores como Artino (2007), señalaron que los estudios sobre procesos de autorregulación del aprendizaje en modalidades de educación en línea, por más de tres décadas se enfocaron en analizar el vínculo entre las creencias motivacionales del estudiante y las estrategias que aplica en su trayecto formativo, e identificar los elementos del proceso de instrucción asociados con la satisfacción y logro de metas de aprendizaje en estos entornos.

En procesos de investigación actual en el contexto de las IES, prevalece la tendencia por encontrar nuevas formas de diseñar la instrucción bajo modelos flexibles de enseñanza que promuevan el aprendizaje autónomo en los estudiantes de nivel superior. De este modo han tomado auge los estudios centrados en conocer las ventajas de la implementación de estrategias digitales y propuestas de enfoque de aula invertida, en respuesta a la necesidad de impulsar la participación activa del estudiante en las decisiones sobre su aprendizaje. En este sentido, toma relevancia la evidente asociación entre los conceptos de educación flexible y aprendizaje abierto "con las distintas formas que toma el aprendizaje en y a través de la red, ya que ofrecen un mayor grado de descentralización del acceso al aprendizaje" (Salinas, 2013, p. 61).

En este punto, Salinas (2013) destaca la importancia de reconocer que, un estudiante de nivel superior, como persona adulta, conoce sus necesidades y limitaciones y ejerce cierta autonomía con respecto a su proceso de aprender. Por tanto, el hecho de incorporar tecnología en los procesos de instrucción, se enmarca dentro de lo que el autor identifica como aprendizaje abierto, enseñanza flexible, aprendizaje en red, y el PLE. Lo cual "significa pues, que el usuario tiene elección de acceso a los recursos de aprendizaje, tiene libertad de maniobra, tiene control activo sobre la forma en que aprende" (p. 62).

Con lo descrito se reafirma la conceptualización del PLE en el campo de la Tecnología Educativa como una estrategia pedagógica centrada en el alumno, que promueve su participación activa en los procesos formativos, al permitirle ejercer una toma de decisiones sobre qué recursos tecnológicos utilizar para crear y obtener información e interactuar socialmente con sus pares mediante sistemas o aplicaciones de la Web 2.0.

La implementación del PLE en el diseño de la instrucción gestionada en ambientes virtuales, se basa en los principios teóricos que explican el proceso de aprendizaje autorregulado, debido a que, desde el punto de vista científico, es importante conocer los pensamientos, sentimientos y acciones que genera una persona de manera cíclica y planificada para lograr sus metas de aprendizaje (Schunk y Zimmerman, 1994). Sobre esta base y de acuerdo a Araujo y Dájer, (2019), el PLE implica "una perspectiva de cómo se pueden relacionar la tecnología y el aprendizaje", con el propósito de desarrollar las habilidades de metacognición y autorregulación en el estudiante dentro de un proceso formativo 
mediado en la utilización de herramientas digitales y plataformas sociales para el trabajo colaborativo, debates y retroalimentación, además de labores de tutoría.

Sin embargo, para lograr tales propósitos, resulta necesario planificar el diseño de la instrucción desde una perspectiva pedagógica que guíe su implementación con propósitos formativos formales y de aprendizaje a lo largo de la vida (Leiva, Cabero y Ugalde, 2018). Por lo que, para efecto de lograr una aproximación conceptual del término PLE, el paradigma sociocognitivo del aprendizaje, que integra diversas teorías de corte cognitivo y social, se estableció como un marco referencial para fundamentarla como una estrategia pedagógica asociada con el desarrollo de habilidades metacognitivas de autorregulación y autonomía que permiten al estudiante autogestionar su aprendizaje en un entorno virtual. Por su parte, Herrera (2020) señala que el constructivismo social resulta clave para explicar la relación entre el PLE y el contexto social en el cual funciona. Dado que, como tal, el constructivismo social se enfoca en analizar los procesos de aprendizaje que se desarrollan en un sistema social donde las personas comparten y aprenden a utilizar artefactos, medios de la cultura y herramientas para aprender. De esta forma, el PLE se establece en el ámbito educativo como una estrategia que integra estrategias-mecanismos cognitivos- y actitudes que promueven el aprendizaje autónomo y autorregulado por parte de las personas (Castañeda y Adell, 2013). Con ello, este enfoque de aprendizaje posibilita que las personas continúen fortaleciendo su formación profesional y el desarrollo de competencias digitales como una condición necesaria para adaptarse a los cambios que surgen en el contexto de la transformación de la sociedad del conocimiento.

\section{MÉTODO}

La puesta en marcha del Plan de Estrategia Digital en una universidad pública del Noreste de México, como respuesta para resolver las eventualidades surgidas en el contexto de contingencia sanitaria por contagio de COVID-19, dio la pauta para que, en una escuela de negocios se resolviera la necesidad de conocer nuevos enfoques pedagógicos para apoyar los procesos de enseñanza-aprendizaje a distancia de los estudiantes que cursan un programa de licenciatura de administración. Por tal razón, este estudio se propuso conocer desde el enfoque del PLE como una pedagogía emergente que integra componentes (estrategias, procesos y tecnologías), que utiliza el estudiante para desarrollar sus habilidades metacognitivas de autogestión del aprendizaje, para cumplir los propósitos de formación formal.

\subsection{Instrumento diseñado}

Para realizar el levantamiento de la información se elaboró un instrumento en formato de cuestionario cerrado, para obtener datos sobre el conocimiento y frecuencia de uso de las herramientas tecnológicas y recursos Web 2.0, que utiliza el estudiante de licenciatura para gestionar sus procesos de aprendizaje de acuerdo a las dimensiones que integra la configuración de su PLE.

El diseño del cuestionario tomó como base los elementos que refieren Castañeda y Adell (2013), y las aportaciones de Castañeda, Prendes y Gutiérrez (2015), para conocer el nivel de competencia del estudiante en el uso de recursos tecnológicos, cómo organiza y selecciona estos recursos, y cuáles utiliza con mayor frecuencia para lograr sus metas de aprendizaje.

El cuestionario compuesto por 13 reactivos, se administró previamente a un grupo de 14 estudiantes del mismo programa de licenciatura como prueba piloto para evaluar su grado de confiabilidad. La organización de los reactivos del cuestionario siguió la categorización de las dimensiones que comprenden la configuración del PLE. Las cuales corresponden a las siguientes: Dimensión1: Estrategias para ordenar y gestionar información; Dimensión 2: Estrategias de autorregulación para cumplir metas, y, Dimensión 3: Estrategias de actuación y reflexión que hacen posible el desarrollo de habilidades metacognitivas (ver tabla 1). 


\subsection{Muestra}

La muestra se conformó por un grupo de 42 estudiantes de Licenciatura en Administración que cursan el décimo semestre, durante el semestre enero-junio de 2021, en modalidad de aula virtual con el soporte de la plataforma MS Teams.

Para seleccionar el tipo de muestra se eligió una muestra intencional o selectiva para cumplir los propósitos de una investigación que tiene el interés en aplicar entrevistas estructuradas a informantes clave que proporcionen la información pertinente sobre los indicadores que se exploran (Cortés, 2012). Por lo cual, los criterios para integrar

Tabla 1 Dimensiones que comprenden el cuestionario y descripción de sus reactivos.

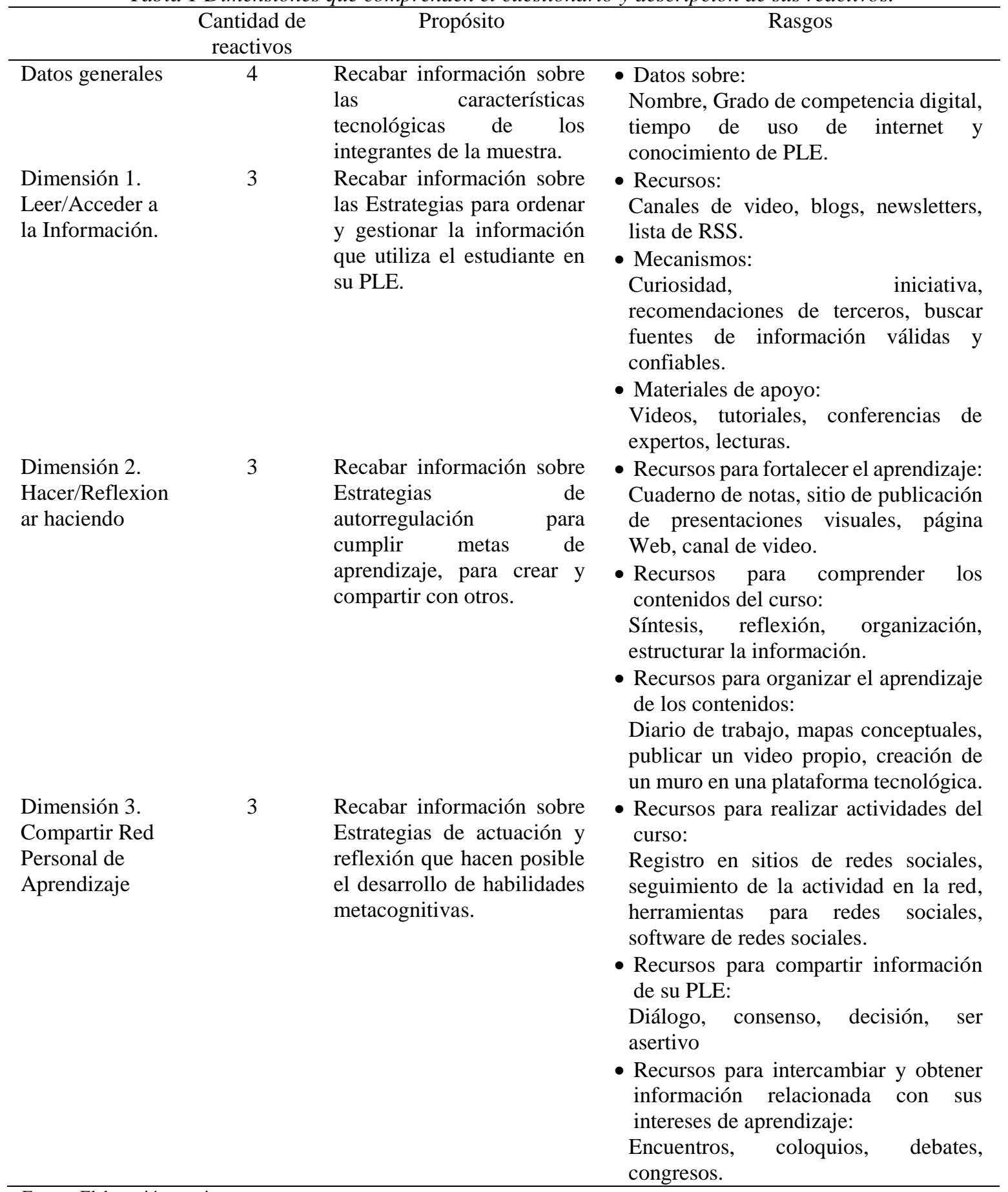

Fuente: Elaboración propia. 
la muestra de participantes fueron los siguientes: ser estudiantes inscritos en una asignatura del décimo semestre de la licenciatura en Administración gestionada en modalidad virtual en la plataforma MS Teams; $\mathrm{y}$, contar con la autorización previa del titular de la asignatura para realizar la invitación a los estudiantes para colaborar en el estudio y dar seguimiento al proceso de aplicación del cuestionario cotejando la lista de participantes del curso.

\section{RESULTADOS}

El cuestionario se divide en 4 partes, la primera parte incluye preguntas generales y las otras 3 partes relacionadas con las dimensiones que configuran el PLE de los estudiantes. La primera dimensión incluye la de lectura y acceso a la información, la segunda dimensión trata sobre lo que hacen los estudiantes y la reflexión que realizan para fortalecer su aprendizaje y, por último, la tercera dimensión que incluye temas de trabajo colaborativo y compartir información.

Se encontró que, respecto al uso de los recursos tecnológicos de última generación utilizados por los estudiantes, todos se consideran competentes, pero vale la pena mencionar que el $12 \%$ se considera extremadamente competente y el $69 \%$ muy competente. Más de la mitad, es decir, el 52\% de ellos invierten entre 6 y 10 horas diarias en internet y el $21 \%$ invierte más de 10 horas para navegar en internet. En cuanto al conocimiento de los entornos personales de aprendizaje (PLE), solo el $71 \%$ mencionó conocer el concepto.

Dentro de los resultados respecto a la dimensión 1 se identificó que el $45 \%$ utilizan canales de video para cumplir con las actividades de lectura y acceso a la información y el 31\% utilizan los Blogs, el resto utilizan otros recursos como libros de texto, listas de RSS y webinars. El 50\% de los estudiantes coinciden en que lo que más les motiva a realizar la búsqueda de información es la curiosidad, seguido de un $38 \%$ de ellos que mencionan que es la búsqueda de fuentes de información válidas y confiables, el 10\% lo hacen por iniciativa propia y el $2 \%$ por recomendaciones de terceros. Para profundizar en la lectura de materiales de apoyo para realizar sus actividades el $52 \%$ utiliza videos, el $21 \%$ lecturas, el $14 \%$ conferencias y el $12 \%$ tutoriales.

Los resultados de la dimensión 2 arrojaron que el $48 \%$ utilizan cuaderno de notas y el $29 \%$ páginas web. Para reflexionar sobre los contenidos, el $45 \%$ estructura su información, el $29 \%$ realiza una reflexión, el $12 \%$ organiza y el $10 \%$ sintetiza la información. Las actividades principales que realizan para organizar el aprendizaje de los contenidos es elaborar mapas conceptuales y la realización de un diario de trabajo con un $40 \%$ y un $29 \%$ de las menciones respectivamente.

El compartir información y trabajar colaborativamente son los temas que cubre la dimensión 3. Los estudiantes utilizan las redes sociales y las actividades en la red para compartir la información y trabajar colaborativamente (93\% de las menciones). El mecanismo preferido para comunicarse es el diálogo $(81 \%)$. Los eventos que utilizan para intercambiar y obtener información relacionada con sus intereses de aprendizaje son los debates con el $38 \%$ de menciones, seguido de los encuentros (21\%), y los congresos (19\%).

\section{CONCLUSIONES}

Este estudio se enfocó en identificar los componentes del PLE de los estudiantes. Como se muestra en los resultados, todos los estudiantes se consideran competentes, lo que confirma que forman parte de los nativos digitales que como comentan CaberoAlmenara et al. (2020) "poseen conocimientos $\mathrm{y}$ habilidades que les permiten manejar las herramientas de las tecnologías de la información y las comunicaciones (TIC) de una manera "natural" y prácticamente sin esfuerzo" (p. 797).

Pérez (2019), haciendo referencia a Botto y Spensieri, 2018, menciona que para los nativos digitales el internet es su principal fuente de información y la utilizan 
cotidianamente, lo cual concuerda con el hallazgo de esta investigación que indica que más de la mitad pasan un tiempo superior a 6 horas en internet existiendo un $21 \%$ que invierte más de 10 horas al día. Sin embargo, a pesar de que sean nativos digitales y pasen gran parte del tiempo con las tecnologías, esa exposición tecnológica no implica capacidad de utilizarla para el aprendizaje (CaberoAlmenara et al., 2020).

Se puede observar que más de la mitad de los estudiantes utilizan el video para cumplir con sus actividades y profundizar en su aprendizaje. Esto concuerda con los resultados del estudio longitudinal que ha realizado Jane Hart desde el 2007 y que publica años tras año en la página https://www.toptools4learning.com/

mostrando las principales herramientas para el aprendizaje siendo en el 2020 y por 5 años consecutivos el YouTube la herramienta número uno tanto para el aprendizaje personal así como también una herramienta para la educación.

Zimmerman (2009) presenta un modelo de fase cíclica de retroalimentación autorreguladora que incluye: la previsión, el desempeño y la autorreflexión. La previsión trata sobre procesos para actuar y preparar el escenario y eso implica fuentes de motivación entre las que se encuentra el interés. Lo presentado por Zimmerman sustenta los resultados obtenidos en el estudio ya que muestra que la mitad de los estudiantes indicaron que lo que más les motiva a realizar la búsqueda de información es la curiosidad, es decir, lo que les lleva a actuar y prepararse para su aprendizaje.

El hecho de que cerca de la mitad de los estudiantes participantes en este estudio mencionaron que utilizan cuaderno de notas, coincide con los resultados de la investigación de Prendes et al. (2019) que muestra que la mayoría prefiere documentos con contenidos de carácter tradicional prefiriendo versiones en papel a la hora de estudiar e incluso los estudiantes continúan tomando notas en papel.

Del mismo modo, los resultados de Prendes et al. (2019) en cuanto a la comunicación coincide con los obtenidos en esta investigación ya que en ambos estudios un porcentaje alto usa las redes sociales, aunque no necesariamente como herramienta académica.

Innumerables tecnologías han surgido a lo largo de los años y la industria 4.0 ha puesto de relieve algunas como la realidad virtual y aumentada, los chatbots y la analítica de datos por mencionar algunas. Sin embargo, aunque la tecnología es un gran apoyo para el proceso de enseñanza-aprendizaje, principalmente en tiempos de pandemia, por sí sola no transformará la educación. Según Sharples (2019) haciendo referencia a Tamin, et al., 2011, menciona que un análisis de 40 años de investigación sobre el impacto de la tecnología educativa en el rendimiento académico demostró solo un efecto de pequeño a moderado, por lo que propone investigaciones futuras en las que se explore buenas combinaciones de tecnología y pedagogía, ya que los éxitos detectados provienen de su uso efectivo en el aula.

En el mismo sentido, Gallego y Chaves (2014) proponen más investigaciones empíricas que asocie el análisis del PLE con la mejora del aprendizaje, así como la capacitación que tienen los docentes y estudiantes en las herramientas que pueden utilizar para conformar sus PLE. Por otro lado, también consideran importante analizar el reto que tienen los docentes de poner en acción el PLE en su práctica didáctica. 


\section{REFERENCIAS}

Araujo, A. y Dájer, M. (diciembre, 2020). Entornos personales de aprendizaje: modelo teórico para el desarrollo metacognitivo. RHS. Revista Humanismo y Sociedad, 8(2). 54-70. https://doi.org/10.22209/rhs.v8n2a04

Artino, A. (junio, 2007). Self-Regulated Learning in Online Education: A Review of the Empirical Literature. International Journal of Instructional Technology \& Distance Education,6(6). En https://www.itdl.org/Journal/Jun_07/article01.htm

Attwell, G. (2007). Personal Learning Environments-the future of eLearning. Elearning papers, 2(1), $1-8$.

Boulahrouz, M., Medir, R. y Calabuig, S. (2019). Tecnologías digitales y educación para el desarrollo sostenible. Un análisis de la producción científica. Pixel-Bit Revista de Medios y Educación, (54). 83-105. https://doi.org/10.12795/pixelbit.2019.i54.05

Cabero, J. y Llorente, M. (2015). Entornos Personales de Aprendizaje (PLE): valoración educativa a través de expertos. Areté, Revista Digital del Doctorado en Educación de la Universidad Central de Venezuela, (1)1. 7-19. En https://dialnet.unirioja.es/servlet/articulo?codigo $=6329677$

Cabero, J. y Llorente, M. (2020). Covid-19: transformación radical de la digitalización en las instituciones universitarias. Revista Campus Virtuales, 9(2). 25-34. En http://www.uajournals.com/ojs/index.php/campusvirtuales/article/view/713/410

Cabero-Almenara, J., Barroso-Osuna, J. y Martínez-Pérez, S. (2020). Estudiantes ¿nativos digitales o residentes y visitantes digitales? Opción: Revista de Ciencias Humanas y Sociales, 93(2), 796-820. En https://dialnet.unirioja.es/servlet/articulo?codigo=7820378

Castañeda, L. y Adell, J. (Eds.). (2013). Entornos Personales de Aprendizaje: claves para el ecosistema educativo en red. En http://hdl.handle.net/10201/30427

Castañeda, L., Prendes, M. y Gutiérrez, I. (2015). Pedagogías emergentes: tecnologías para la educación flexible. En J. Cabero y J. Barroso. (Coords.). Nuevos retos en tecnología educativa. (pp. 195-214). España: Editorial Síntesis.

Chaves, E. y Sola, T. (enero-abril, 2018). Entornos personales de aprendizaje (PLE) en el Grado de Educación Primaria de la Universidad de Granada. Revista Electrónica Educare,22(1), 1-18. http://dx.doi.org/10.15359/ree.22-1.12

Chiecher, A. y Melgar, M. (2018). ¿Lo saben todo? Innovaciones educativas orientadas a promover competencias digitales en universitarios. Apertura, 10(2). 110-123. https://doi.org/10.32870/ap.v10n2.1374

Cortés, M. (2012). Metodología de la investigación. Características del conocimiento científico. Preguntas de investigación. Técnicas e instrumentos para recabar información. México: Trillas.

Dabbagh, N., y Kitsantas, A. (2012). Personal Learning Environments, social media, and selfregulated learning: A natural formula for connecting formal and informal learning. The Internet and Higher Education, 15(1), 3-8. https://doi.org/10.1016/j.iheduc.2011.06.002

Downes, S. (2007). Learning networks in practice. Emerging technologies for learning, 2(4), 20. En https://d1wqtxts1xzle7.cloudfront.net/30807230/8913424-with-coverpage.pdf?Expires=1621042583\&Signature=M 1YA6TgiZBsf4GT3CcbkKR9zf5CTsbNQu 6M5zXcEHR2RZQdPfQ0JQwcQrfMMm0D EqMEoRje0GKOvc UhphAsK gzgUVlpLs cg9wtRzLM3iWU7y6m-

zxv1OMohCoGsb1HaCSeZZpf9HccRtLSIDu2XdTbnDiUqSRUiXgxj3r9fKe6olR61javvjf N4a1eJLRiC1v-LYDjNUSIHryOzWQIc-2-

4aN4qMBGAwEfmEnfpBSmpFb3JUTah1QZImcXPMsy6scBPUK2y6ujMgSgeKeVJyJwZ UCXKSvzFb5kJtyEccIRCCcu1ihcrk25ZBEyYZ vyD5bahWEUKuebNPsxJyg_\&KeyPair-Id=APKAJLOHF5GGSLRBV4ZA

Gallego, M. y Chaves, E. (septiembre, 2014). Tendencias en estudios sobre Entornos Personales de Aprendizaje (Personal Learning Environments -PLE-). Edutec, Revista Electrónica de 
Tecnología Educativa, (49). 1-22. https://doi.org/10.21556/edutec.2014.49.89

García, J. y González, M. (diciembre, 2019). Cómo generan y gestionan contenidos los estudiantes de educación de Costa Rica: una contribución al estudio de su entorno personal de aprendizaje. Digital Education Review, (36). 15-35. En https://dialnet.unirioja.es/servlet/articulo?codigo $=7282526$

Hernández, M. A. (2016). Gestión del conocimiento, actividad científica y entornos personales de aprendizaje (PLEs): una bibliometría de la PLE Conference. Edutec. Revista Electrónica de Tecnología Educativa, O(55), 329.

Herrera, C. (2019). Monografía: propuesta de entorno personal de aprendizaje (ple) virtual para el desarrollo de competencias tecnológicas y uso en educación superior (Tesis de maestría). Recuperada de: http://hdl.handle.net/10882/9686.

Jerez, Y. y Barroso, J. (2020). Identificación de los componentes del Entorno Personal de Aprendizaje de estudiantes de ingeniería. Revista de Educación mediática y TIC, EDMETIC, 9(2), 202221. https://doi.org/10.21071/edmetic.v9i2.12602

Leiva, J., Cabero, J. y Ugalde, L. (2018). Entornos personales de aprendizaje (PLE) en estudiantes universitarios de Pedagogía. Revista Latinoamericana de Tecnología Educativa, 17(1). En http://dx.medra.org/10.17398/1695-288X.17.1.25

López, P., González, V., Aguiar, M. y Artiles, J. (2017). La gestión de la información en entornos personales de aprendizaje: estudio exploratorio en alumnado de último curso de grado. Revista Complutense de Educación, 28(4). 1303-1320. http://dx.doi.org/10.5209/RCED.51849

González, Z. y Calvo, M. (2019). Entorno Personal de Aprendizaje Autónomo 2.0 en el Grado de Administración y Dirección de Empresas. En De los procesos de cambio al cambio con sentido. (Coord. Ana Vega Navarro). Servicio de Publicaciones Universidad de la Laguna. 185-200. https://doi.org/10.25145/b.innovaull.2019

Meza, J., Morales, M. y Flores, R. (2016). Variables individuales relacionadas con la instrucción en el uso de entornos personales de aprendizaje. Educación [online], 25(48). 87-106. http://dx.doi.org/10.18800/educacion.201601.005.

Milligan, C., Beauvoir, P., Johnson, M., Sharples, P., Wilson, S. y Liber, O. (2006). Developing a reference model to describe the personal learning environment. En W. Nejdl and K. Tochtermann (Eds.), European Conference on Technology Enhanced Learning, (pp. 506 511). Berlín, Heidelberg: Springer-Verlag. En https://www.researchgate.net/profile/ColinMilligan/publication/221549636_Developing_a_Reference_Model_to_Describe_the_Perso nal_Learning_Environment/links/02e7e53591985f0932000000/Developing-a-ReferenceModel-to-Describe-the-Personal-Learning-Environment.pdf

Ortiz, A., Moroto, J. y Agreda, M. (2017). Uso y Recursos Tecnológicos de los Entornos Personales de Aprendizaje con Estudiantes de los Grados de Maestro en Educación Infantil y Primaria. Formación Universitaria, 10(5). 41-49. http://dx.doi.org/10.4067/S071850062017000500005

Pane, J. F., Steiner, E. D., Baird, M. D., y Hamilton, L. S. (2015). Continued Progress: Promising Evidence on Personalized Learning. En https://www.rand.org/pubs/research_reports/RR1365.html.

Pérez, J. (2019). DINADI: una estrategia para el diagnóstico de nativos digitales en el ámbito universitario. Revista Paradigma, 40(1), 56-75. En http://revistaparadigma.online/ojs/index.php/paradigma/article/view/719

Prendes, M., Román, M. y González, V. (2019). How university students use technologies to learn: a survey about PLE un Spain. Education in the Knowledge Society, 20, 10.1-10.12. https://doi.org/10.14201/eks2019_20_a10

Recio, F., Silva, J., \& Abricot, N. (2020). Analysis of the Digital Competence in the Initial Formation of University Students: A Meta-Analysis Study on the Web of Science. Pixel Bit Revista de Medios y Educación, (59), 125-146. https://doi.org/10.12795/pixelbit.77759 
Salinas, J. (2013). Enseñanza flexible y aprendizaje abierto, fundamentos clave de los PLEs.

Capítulo 3. En Castañeda, L. y Adell, J. (Eds.). (2013). Entornos Personales de Aprendizaje: claves para el ecosistema educativo en red. Alcoy: Marfil.

Schunk, D. H. y Zimmerman, B. J. (1994). Prefacio. En D. H. Schunk \& B. J. Zimmerman (Eds.), Self-regulation of learning and performance: Issues and educational applications (pp. ixxi). Hillsdale, NJ: Erlbaum.

Sharples, M. (2019). Visions for the Future of Educational Technology. En Ferguson, R., Jones, A. y Scanlon, E. (Eds.), Educational Visions: Lessons from 40 years of innovation. Pp. 151-166. London: Ubiquity Press. https://doi.org/10.5334/bcg.j.

Tarancón, B. (2017). Implementación de un modelo de entorno personal de aprendizaje en el Grado en Educación (Infantil y Primaria). Estudio de caso. Revista de Educación Mediática y TIC, EDMETIC, 6(2). 232-254. https://doi.org/10.21071/edmetic.v6i2.6933

UNESCO. (2020). Educación para el desarrollo sostenible. Hoja de ruta. En https://unesdoc.unesco.org/ark:/48223/pf0000374896?posInSet=6\&queryId=53f99e0b6ceb-461f-8a8c-31795a772e94

Zimmerman, B. J., \& Cleary, T. J. (2009). Motives to self-regulate learning: A social cognitive account. En K. R. Wenzel y A. Wigfield (Eds.), Educational psychology handbook series. Handbook of motivation at school (p. 247-264). Routledge/Taylor \& Francis Group. 